\title{
CALDERÓN-ZYGMUND OPERATORS AND COMMUTATORS ON WEIGHTED LORENTZ SPACES
}

\author{
MARÍA J. CARRO*, HONGLIANG LI $^{\dagger}$, JAVIER SORIA*, AND QINXIU SUN ${ }^{\ddagger}$
}

ABstract. We find necessary conditions (which are also sufficient, for some particular cases) for a pair of weights $u$ and $w$ such that a Calderón-Zygmund operator $T$, or its commutator $[b, T]$, with $b \in B M O$, is bounded on the weighted Lorentz spaces $\Lambda_{u}^{p}(w)$, for $1<p<\infty$. This result completes the study already known for the Hardy-Littlewood maximal operator and the Hilbert transform, and hence unifies the weighted theories for the $A_{p}$ and $B_{p}$ classes.

Dedicated to our mentor, tutor and, above all, dear friend Guido Weiss, thanks to whom we are a little "weisser".

\section{INTRODUCTION}

The main goal of this work is to complete the unification of classical results about the boundedness of Calderón-Zygmund operators $T$ on different weighted settings, like the Lebesgue spaces $L^{p}(u)$ or the Lorentz spaces $\Lambda^{p}(w)$, into a general framework involving both theories. This is done by considering the so-called weighted Lorentz spaces $\Lambda_{u}^{p}(w)$ defined as the set of measurable functions $f: \mathbb{R}^{n} \rightarrow \mathbb{R}$ for which (see Section 2 for the rigorous definition and the meaning of the different notations):

$$
\|f\|_{\Lambda_{u}^{p}(w)}=\left(\int_{0}^{\infty} f_{u}^{*}(t)^{p} w(t) d t\right)^{1 / p}<\infty .
$$

Recall that, if $w=1$, then $\Lambda_{u}^{p}(w)=L^{p}(u)$ and if $u=1$, then $\Lambda_{u}^{p}(w)=\Lambda^{p}(w)$.

It is well-known [7] that, for this kind of singular operators, the boundedness of $T: L^{p}(u) \rightarrow L^{p}(u)$ holds if $u$ is in the $A_{p}$ class of weights, $1<p<\infty$ :

$$
u \in A_{p} \Longleftrightarrow \sup _{\{Q: Q \text { is a cube }\}}\left(\frac{1}{|Q|} \int_{Q} u(x) d x\right)\left(\frac{1}{|Q|} \int_{Q} u^{-1 /(p-1)}(x) d x\right)^{p-1}<\infty .
$$

For the case $p=\infty$, we define $A_{\infty}=\cup_{p>1} A_{p}$. The $A_{p}$ class was introduced by Muckenhoupt [14] and characterized as follows: for every $p>1$,

$$
M: L^{p}(u) \longrightarrow L^{p}(u) \quad \Longleftrightarrow u \in A_{p},
$$

where $M$ is the Hardy-Littlewood maximal operator

$$
M f(x)=\sup \left\{\frac{1}{|Q|} \int_{Q}|f(y)| d y: Q \text { is a cube in } \mathbb{R}^{n} \text { and } x \in Q\right\} .
$$

2010 Mathematics Subject Classification. 46E30, 46B42.

Key words and phrases. Calderón-Zygmund operators, commutators, weighted Lorentz spaces.

*M. J. Carro and J. Soria have been partially supported by the Spanish Government grant MTM2016-75196-P (MINECO/FEDER, UE) and Grupo UCM-970905.

${ }^{\dagger}$ H. Li has been supported by the National Natural Science Foundation of China (11961056).

${ }^{\ddagger}$ Q. Sun has been supported by the by the Natural Science Foundation of Zhejiang Province of China (LY19A010001). 
Moreover, in the case $T=H$, the Hilbert transform,

$$
H f(x)=\mathrm{p} . \mathrm{v} \cdot \int_{-\infty}^{\infty} \frac{f(y)}{x-y} d y=\lim _{\varepsilon \rightarrow 0^{+}} \int_{|x-y|>\varepsilon} \frac{f(y)}{x-y} d y,
$$

it was proved in [11] that

$$
H: L^{p}(u) \longrightarrow L^{p}(u) \quad \Longleftrightarrow u \in A_{p} .
$$

If we now consider the case of the weighted Lorentz spaces $\Lambda^{p}(w)$, Neugebauer proved in $[15]$ that

$$
H: \Lambda^{p}(w) \rightarrow \Lambda^{p}(w) \text { if and only if } w \in B_{\infty}^{*} \cap B_{p},
$$

for all $1<p<\infty$, where the Ariño-Muckenhoupt $B_{p}$ class is characterized [3] by the condition

$$
r^{p} \int_{r}^{\infty} \frac{w(t)}{t^{p}} d t \leq C \int_{0}^{t} w(s) d s, \quad \text { for all } r>0,
$$

and the $B_{\infty}^{*}$ class is defined as

$$
\int_{0}^{t} \frac{W(s)}{s} d s \leq C W(t), \quad \text { with } W(t)=\int_{0}^{t} w(s) d s .
$$

It is well-known that there are many different characterizations of the $B_{p}$ class (see, e.g., $[3,5,17])$. In particular, (3) is equivalent to the boundedness of

$$
A: L_{\mathrm{dec}}^{p}(w) \rightarrow L^{p}(w),
$$

where $A$ is the Hardy operator defined by

$$
A f(x)=\frac{1}{x} \int_{0}^{x} f(t) d t, x>0
$$

and $L_{\mathrm{dec}}^{p}(w)$ is the cone of all non-increasing functions in $L^{p}(w)$ [3]. Hence, since the decreasing rearrangement (see Section 2 for the definition) of $M$ satisfies that [4, Theorem III.3.8]

it immediately holds that

$$
(M f)^{*}(t) \approx \frac{1}{t} \int_{0}^{t} f^{*}(s) d s
$$

$$
w \in B_{p} \quad \Longleftrightarrow \quad M: \Lambda^{p}(w) \longrightarrow \Lambda^{p}(w) .
$$

A simple remark that we will need later on is that the primitive of a $B_{p}$-weight satisfies the $\Delta_{2}$-condition; namely, if $w \in B_{p}$, then

$$
W(2 t) \leq C W(t), \quad \text { for every } t>0 .
$$

On the other hand, the condition $w \in B_{\infty}^{*}$ is equivalent [15] to the boundedness of

$$
A^{*}: L_{\mathrm{dec}}^{p}(w) \rightarrow L^{p}(w),
$$

with $A^{*}$ the adjoint Hardy operator

$$
A^{*} f(x)=\int_{x}^{\infty} f(t) \frac{d t}{t}, x>0
$$

Hence, condition (2) follows from the inequality [4, Theorem III.4.8]

$$
(H f)^{*}(t) \lesssim \frac{1}{t} \int_{0}^{t} f^{*}(s) d s+\int_{t}^{\infty} f^{*}(s) \frac{d s}{s}
$$


as well as the converse inequality [4, Proposition III.4.10]. Taking into considerations the equivalences (1) and (2), it is a natural question to characterize the weights $u$ and $w$ so that

$$
M, H, T: \Lambda_{u}^{p}(w) \longrightarrow \Lambda_{u}^{p}(w)
$$

are bounded operators. The complete answer for the operator $M$ (see Section 2 for the definitions involved) was given in [5, Theorem 3.3.5]: for every $p>1$,

$$
M: \Lambda_{u}^{p}(w) \longrightarrow \Lambda_{u}^{p}(w) \quad \Longleftrightarrow w \in B_{p}(u),
$$

where $w \in B_{p}(u)$ if there exists $q \in(0, p)$ such that, for every finite family of cubes $\left(Q_{j}\right)_{j=1}^{J}$ and every family of measurable sets $\left(E_{j}\right)_{j=1}^{J}$, with $E_{j} \subset Q_{j}$, for every $j$, we have that

$$
\frac{W\left(u\left(\bigcup_{j} Q_{j}\right)\right)}{W\left(u\left(\bigcup_{j} E_{j}\right)\right)} \leq C \max _{j}\left(\frac{\left|Q_{j}\right|}{\left|E_{j}\right|}\right)^{q} .
$$

Finally, the solution for the Hilbert transform $H$ can be found in [1]: for every $p>1$,

$$
H: \Lambda_{u}^{p}(w) \longrightarrow \Lambda_{u}^{p}(w) \quad \Longleftrightarrow u \in A_{\infty}, w \in B_{\infty}^{*} \text {, and } w \in B_{p}(u) .
$$

It is worth mentioning that, contrary to the case of the Hilbert transform, the boundedness of $M$ on $\Lambda_{u}^{p}(w)$ does not imply that $u \in A_{\infty}$ (there are examples for which $u$ may not even satisfy the doubling condition [5, Theorem 3.3.10]).

The main result of this paper is to prove that these conditions also extend to Calderón-Zygmund operators $T$. Since they are also necessary for the case $T=H$, we see that they cannot be improved, for a general $T$, and hence we fully characterize (4) in this setting.

Theorem 1.1 (Main result). Let $1<p<\infty$ and let $T$ be a Calderón-Zygmund operator. Then, if $u \in A_{\infty}, w \in B_{\infty}^{*}$, and $w \in B_{p}(u)$, we have that

$$
T: \Lambda_{u}^{p}(w) \longrightarrow \Lambda_{u}^{p}(w)
$$

We will also obtain the analogous result for the corresponding $B M O$ commutator:

Theorem 1.2. Let $b \in B M O\left(\mathbb{R}^{n}\right)$ and let $T$ be a Calderón-Zygmund operator. Then, if $u \in A_{\infty}, w \in B_{\infty}^{*}$, and $w \in B_{p}(u)$, we have that

$$
\|[b, T](f)\|_{\Lambda_{u}^{p}(w)} \leq C\|b\|_{B M O}\|f\|_{\Lambda_{u}^{p}(w)} .
$$

Remark 1.3. If $w=1$ and $p>1$, it holds that $w \in B_{p}(u)$ is equivalent to $u \in A_{p}$, and if $u=1$, then $B_{p}(1)=B_{p}$. Thus, Theorem 1.1 recovers the previous results on $L^{p}(u)$ and $\Lambda^{p}(w)$ spaces $[11,1]$ and unifies the classical theories on $A_{p}$ and $B_{p}$ weights.

The paper is organized as follows: In Section 2 we will present all the definitions we need and some technical lemmas, and Section 3 is devoted to the proofs of the main results.

As usual the symbol $A \lesssim B$ will be used to indicate that there exists a positive constants $C$ independent of all parameters involved so that $A \leq C B$; similarly, $A \approx B$ means that $A \lesssim B$ and $B \lesssim A$. 


\section{Previous definitions and technical lemmas}

Functions $u: \mathbb{R}^{n} \rightarrow \mathbb{R}_{+}$and $w: \mathbb{R}_{+} \rightarrow \mathbb{R}_{+}$are called weights whenever they are Lebesgue measurable functions, positive, not identically zero, and locally integrable. If $w$ is a weight on $\mathbb{R}_{+}$, we denote by $W(t)=\int_{0}^{t} w(s) d s$ its primitive, and observe that $W(t)<\infty$, for all $t>0$.

Given a weight $u$ in $\mathbb{R}^{n}$ and a measurable function $f$, its decreasing rearrangement $f_{u}^{*}$ is defined as [4]

$$
f_{u}^{*}(t)=\inf \left\{s: \lambda_{f}^{\mu}(s) \leq t\right\}, t \geq 0,
$$

where

$$
\lambda_{f}^{u}(s)=u(\{x \in X:|f(x)|>s\}), s \geq 0
$$

is the distribution function of $f$ with respect to the measure $u(x) d x$. In particular, we write $f_{u}^{*}=f^{*}$ and $\lambda_{f}^{u}=\lambda_{f}$, if $u=1$.

Definition $2.1([6,5])$. Let $u$ be a weight in $\mathbb{R}^{n}$ and $w$ a weight in $\mathbb{R}_{+}$. For every $p>1$, the weighted Lorentz space $\Lambda_{u}^{p}(w)$ is the class of all measurable functions $f$ such that

$$
\|f\|_{\Lambda_{u}^{p}(w)}=\left(\int_{0}^{\infty} f_{u}^{*}(t)^{p} w(t) d t\right)^{1 / p}<\infty .
$$

As we have already observed, with $u=1, \Lambda_{1}^{p}(w)=\Lambda^{p}(w)$ is the weighted Lorentz space [13]; if $u=1$ and $w(t)=t^{p / q-1}$, then we recover the classical Lorentz spaces $L^{q, p}[18$, Chapter V§3], and with $w=1$,

$$
\begin{aligned}
\|f\|_{\Lambda_{u}^{p}(1)} & =\left(\int_{0}^{\infty} f_{u}^{*}(t)^{p} d t\right)^{1 / p}=\left(p \int_{0}^{\infty} t^{p-1} \lambda_{f}^{u}(t) d t\right)^{1 / p} \\
& =\left(\int_{\mathbb{R}^{n}}|f(x)|^{p} u(x) d x\right)^{1 / p}=\|f\|_{L^{p}(u)} .
\end{aligned}
$$

Definition 2.2. [10, Definition 4.1.2] A function $K(x, y)$ on $\mathbb{R}^{n} \times \mathbb{R}^{n} \backslash\{(x, x): x \in$ $\left.\mathbb{R}^{n}\right\}$ is called a standard kernel if there exists $\delta, A>0$ satisfying the size condition

$$
|K(x, y)| \leq \frac{A}{|x-y|^{n}}
$$

and the regularity condition

$$
\left|K(x, y)-K\left(x^{\prime}, y\right)\right| \leq \frac{A\left|x-x^{\prime}\right|^{\delta}}{\left(|x-y|+\left|x^{\prime}-y\right|\right)^{n+\delta}},
$$

when $\left|x-x^{\prime}\right| \leq \frac{1}{2} \max \left(|x-y|,\left|x^{\prime}-y\right|\right)$ and

$$
\left|K(x, y)-K\left(x, y^{\prime}\right)\right| \leq \frac{A\left|y-y^{\prime}\right|^{\delta}}{\left(|x-y|+\left|x-y^{\prime}\right|\right)^{n+\delta}},
$$

when $\left|y-y^{\prime}\right| \leq \frac{1}{2} \max \left(|x-y|,\left|x-y^{\prime}\right|\right)$. The class of all standard kernels with constants $\delta$ and $A$ is denoted by $S K(\delta, A)$.

Definition 2.3. [10, Definition 4.1.8] Let $0<\delta, A<\infty$ and $K \in S K(\delta, A)$. T is called a Calderón-Zygmund operator associated with the standard kernel $K$ if $T$ is defined on the class of Schwartz functions $\mathcal{S}\left(\mathbb{R}^{n}\right)$, admits a bounded extension on $L^{2}\left(\mathbb{R}^{n}\right)$

$$
\|T f\|_{L^{2}} \leq B\|f\|_{L^{2}}, \quad \text { for all } f \in L^{2}\left(\mathbb{R}^{n}\right)
$$


and

$$
T f(x)=\int_{\mathbb{R}^{n}} K(x, y) f(y) d y,
$$

for all $f \in C_{0}^{\infty}\left(\mathbb{R}^{n}\right)$ and $x \notin \operatorname{supp}(f)$.

We recall that the first example of a Calderón-Zygmund operator is the Hilbert transform. Also, for a locally integrable function $b$, the commutator of $T$ and $b$ is defined as

$$
[b, T](f)=b T(f)-T(b f) .
$$

For a general Calderón-Zygmund operator $T$, it is well-known that $T$ is bounded on $L^{p}\left(\mathbb{R}^{n}\right)$, for all $p \in(1, \infty)[10$, Theorem 4.2.2]. Coifman and Fefferman [7] proved that $T$ is bounded on the weighted Lebesgue space $L^{p}(u)$, when $u \in A_{p}$ and $1<p<\infty$. For the commutator, Coifman, Rochberg and Weiss [8] proved that $[b, T]$ is bounded on $L^{p}\left(\mathbb{R}^{n}\right), 1<p<\infty$, if $b$ is a $B M O\left(\mathbb{R}^{n}\right)$ function. In the sequel, we always assume $b \in B M O\left(\mathbb{R}^{n}\right)$ in the commutator $[b, T]$.

For $\beta>0$, let $M_{\beta}$ be the modified Hardy-Littlewood maximal function

$$
M_{\beta}(f)(x)=M\left(|f|^{\beta}\right)^{1 / \beta}(x)=\left(\sup _{x \in Q} \frac{1}{|Q|} \int_{Q}|f(y)|^{\beta} d y\right)^{1 / \beta},
$$

and let $M_{\beta}^{\sharp}$ be the sharp maximal function

$$
M_{\beta}^{\sharp}(f)(x)=\sup _{x \in Q} \inf _{c}\left(\left.\frac{1}{|Q|} \int_{Q}|| f(y)\right|^{\beta}-|c|^{\beta} \mid d y\right)^{1 / \beta},
$$

where $Q$ is a cube in $\mathbb{R}^{n}$.

The following two lemmas describe some relations among Calderón-Zygmund operators, commutators, the Hardy-Littlewood maximal function and the sharp maximal functions.

Lemma 2.4. [2]. Let $T$ be a Calderón-Zygmund operator and $0<\beta<1$. Then

$$
M_{\beta}^{\sharp}(T(f))(x) \leq C M(f)(x)
$$

for all bounded functions $f$ with compact support.

Lemma 2.5. [16]. Let $T$ be a Calderón-Zygmund operator, $b \in B M O\left(\mathbb{R}^{n}\right)$, and $0<\beta<\varepsilon$. Then

$$
M_{\beta}^{\sharp}([b, T](f))(x) \leq C\|b\|_{B M O}\left(M_{\varepsilon}(T(f))(x)+M^{2}(f)(x)\right),
$$

for all bounded functions $f$ with compact support, where $M^{2}(f)=M(M(f))$.

Lemma 2.6. Let $1<p<\infty, u \in A_{\infty}, w \in B_{\infty}^{*}$, and $W \in \Delta_{2}$. Then

$$
\|M(f)\|_{\Lambda_{u}^{p}(w)} \lesssim\left\|M^{\sharp}(f)\right\|_{\Lambda_{u}^{p}(w)},
$$

if $\|M(f)\|_{\Lambda_{u}^{p}(w)}<\infty$.

Proof. Let us recall the following good- $\lambda$ inequality [9, Lemma 4.2]: if $u \in A_{\infty}$, there exist $C>0$ and $\rho>0$, such that for all $t, \gamma>0$ :

$$
u\left(\left\{x: M(f)(x)>2 t, M^{\sharp}(f)(x) \leq \gamma t\right\}\right) \leq C \gamma^{\rho} u(\{x: M(f)>t\})
$$


for all locally integrable functions $f$ on $\mathbb{R}^{n}$. By [5, Proposition 2.2.5], we know that

$$
\begin{aligned}
\|M(f)\|_{\Lambda_{u}^{p}(w)}^{p} & =\int_{0}^{\infty} p W(u(\{x: M(f)(x)>t\})) t^{p-1} d t \\
& =p 2^{p} \int_{0}^{\infty} W(u(\{x: M(f)(x)>2 t\})) t^{p-1} d t .
\end{aligned}
$$

Since $W \in \Delta_{2}$, then

$$
W(s+t) \leq c(W(s)+W(t)), \text { for all } s, t>0,
$$

and hence, using (8), we obtain

$$
\begin{aligned}
\|M(f)\|_{\Lambda_{u}^{p}(w)}^{p} \leq & p 2^{p} c \int_{0}^{\infty} W\left(u\left(\left\{x: M(f)(x)>2 t, M^{\sharp}(f)(x) \leq \gamma t\right\}\right)\right) t^{p-1} d t \\
& +p 2^{p} c \int_{0}^{\infty} W\left(u\left(\left\{x: M(f)(x)>2 t, M^{\sharp}(f)(x)>\gamma t\right\}\right)\right) t^{p-1} d t \\
\leq & p 2^{p} c \int_{0}^{\infty} W\left(C \gamma^{\rho} u(\{x: M(f)(x)>t\})\right) t^{p-1} d t \\
& +p 2^{p} c \int_{0}^{\infty} W\left(u\left(\left\{x: M^{\sharp}(f)(x)>\gamma t\right\}\right)\right) t^{p-1} d t \\
= & p 2^{p} c \int_{0}^{\infty} W\left(C \gamma^{\rho} u(\{x: M(f)(x)>t\})\right) t^{p-1} d t \\
& +(2 / \gamma)^{p} c\left\|M^{\sharp}(f)\right\|_{\Lambda_{u}^{p}(w)}^{p}
\end{aligned}
$$

To estimate the last integral, we use the following result, which actually gives a characterization of $B_{\infty}^{*}\left[1\right.$, Proposition 2.3 (vi)]: if $w \in B_{\infty}^{*}$, then for every $\varepsilon>0$, there exists an $\alpha>0$ such that $W(t) \leq \varepsilon W(s)$, provided $t \leq \alpha s$. Thus, with $\varepsilon=\left(2^{p+1} c\right)^{-1}$, there exists an $\alpha>0$ such that, if $C \gamma^{\rho} \leq \alpha$, then

$$
\left.W\left(C \gamma^{\rho} u(\{x: M(f)(x)>t\})\right) \leq\left(2^{p+1} c\right)^{-1} W(u(\{x: M(f)(x)>t\}))\right) .
$$

Therefore, taking $\gamma \leq(\alpha / C)^{1 / \rho}$ in (8), we finally obtain

$$
\begin{aligned}
\|M(f)\|_{\Lambda_{u}^{p}(w)}^{p} \leq & p 2^{p} c\left(2^{p+1} c\right)^{-1} \int_{0}^{\infty} W(u(\{x: M(f)(x)>t\})) t^{p-1} d t \\
& +(2 / \gamma)^{p} c\left\|M^{\sharp}(f)\right\|_{\Lambda_{u}^{p}(w)}^{p} \\
= & 2^{-1}\|M(f)\|_{\Lambda_{u}^{p}(w)}^{p}+(2 / \gamma)^{p} c\left\|M^{\sharp}(f)\right\|_{\Lambda_{u}^{p}(w)}^{p} .
\end{aligned}
$$

Since $\|M(f)\|_{\Lambda_{u}^{p}(w)}<\infty$, we conclude $(7)$.

By a homogeneity argument, it is easy to see that (7) also holds for the modified operators $M_{\beta}$ and $M_{\beta}^{\sharp}$.

The following result gives a useful estimate we will need to prove our main theorem:

Lemma 2.7. If $w \in B_{\infty}^{*}$, there exists $\varepsilon>0$ so that

$$
\int_{0}^{1} \frac{W(t)}{t^{1+\varepsilon}} d t<\infty
$$


Proof. Using the hypothesis and integration by parts, we have that, for $0<a<1$,

$$
\begin{aligned}
\int_{a}^{1} \frac{W(t)}{t^{1+\varepsilon}} d t & =\int_{0}^{1} \frac{W(s)}{s} d s-\frac{1}{a^{\varepsilon}} \int_{0}^{a} \frac{W(s)}{s} d s+\varepsilon \int_{a}^{1}\left(\int_{0}^{t} \frac{W(s)}{s} d s\right) t^{-\varepsilon-1} d t \\
& \lesssim \int_{0}^{1} \frac{W(s)}{s} d s+C \varepsilon \int_{a}^{1} \frac{W(t)}{t^{1+\varepsilon}} d t .
\end{aligned}
$$

Taking $\varepsilon>0$ so that $C \varepsilon<1$, we obtain that

$$
\int_{a}^{1} \frac{W(t)}{t^{1+\varepsilon}} d t \leq \frac{1}{1-C \varepsilon} \int_{0}^{1} \frac{W(s)}{s} d s<\infty
$$

and letting $a \rightarrow 0$, we get the result.

\section{Proof of the main Results}

Before we start, let us observe that if $w \in B_{\infty}^{*}$, then $w \notin L^{1}\left(\mathbb{R}_{+}\right)$. This follows easily form the fact that [15, p. 143]

$$
\int_{0}^{r} \log \left(\frac{r}{x}\right) w(x) d x \lesssim \int_{0}^{r} w(x) d x .
$$

Hence,

$$
\infty=\lim _{r \rightarrow \infty} \int_{1}^{2} \log \left(\frac{r}{2}\right) w(x) d x \lesssim \int_{0}^{\infty} w(x) d x .
$$

Thus, we conclude that the class of all bounded functions with compact support is dense in the weighted Lorentz spaces $\Lambda_{u}^{p}(w)[5,12]$, result that we will use in the proofs of main results.

Proof of Theorem 1.1. Let $0<\beta<1$. Since $w \in B_{p}(u)$, its primitive satisfies the doubling condition $W \in \Delta_{2}$ [5, Lemma 3.3.1]. Now, using that $w \in B_{\infty}^{*}$, by Lemma 2.6, Lemma 2.4 and again by condition $w \in B_{p}(u)$, which is equivalent to (5), we get that, if $\left\|M_{\beta} T(f)\right\|_{\Lambda_{u}^{p}(w)}<\infty$, then

$$
\|T(f)\|_{\Lambda_{u}^{p}(w)} \leq\left\|M_{\beta}(T(f))\right\|_{\Lambda_{u}^{p}(w)} \lesssim\left\|M_{\beta}^{\sharp}(T(f))\right\|_{\Lambda_{u}^{p}(w)} \lesssim\|M(f)\|_{\Lambda_{u}^{p}(w)} \lesssim\|f\|_{\Lambda_{u}^{p}(w)} .
$$

Since $w \in B_{p}(u)$ implies $w \in B_{p / \beta}(u)$ [5, Theorem 3.3.5], we have that

$$
\left\|M_{\beta}(T(f))\right\|_{\Lambda_{u}^{p}(w)}=\left\|M\left(|T(f)|^{\beta}\right)\right\|_{\Lambda_{u}^{p / \beta}(w)}^{1 / \beta} \leq C\left\||T(f)|^{\beta}\right\|_{\Lambda_{u}^{p / \beta}(w)}^{1 / \beta}=C\|T(f)\|_{\Lambda_{u}^{p}(w)} .
$$

So, to obtain

$$
\|T(f)\|_{\Lambda_{u}^{p}(w)} \leq C\|f\|_{\Lambda_{u}^{p}(w)}
$$

we only need to show that $\|T(f)\|_{\Lambda_{u}^{p}(w)}<\infty$.

Let $R>0$ so that $\operatorname{supp} f \subset B(0, R)$ and set $B=B(0, R)$. Then

$$
\begin{aligned}
\|T(f)\|_{\Lambda_{u}^{p}(w)} \lesssim & \left(\int_{0}^{\infty} W(u(\{x \in 3 B:|T(f)(x)|>t\})) t^{p-1} d t\right)^{1 / p} \\
& +\left(\int_{0}^{\infty} W\left(u\left(\left\{x \in \mathbb{R}^{n} \backslash 3 B:|T(f)(x)|>t\right\}\right)\right) t^{p-1} d t\right)^{1 / p} \\
= & I_{1}+I_{2} .
\end{aligned}
$$


For $I_{2}$, it is easy to see, by the condition (6) of $T$ that

$$
|T(f)(x)| \leq C M(f)(x),
$$

since if $x \in(3 B)^{c}$ and $y \in B$, we have that

$$
|x| \geq 3 R, \quad|x-y| \geq|x|-R \geq|x| / 2, \text { and } B \subset B(0,|x|) .
$$

Therefore,

$$
|T(f)(x)| \lesssim \int_{B}|x-y|^{-n}|f(y)| d y \lesssim \frac{1}{|x|^{n}} \int_{B(0,|x|)}|f(y)| d y \lesssim M(f)(x),
$$

and hence, since $w \in B_{p}(u)$,

$$
I_{2} \lesssim\|M(f)\|_{\Lambda_{u}^{p}(w)} \lesssim\|f\|_{\Lambda_{u}^{p}(w)}<\infty .
$$

To estimate $I_{1}$, using Lemma 2.7, let us take $r>1$ big enough to have that

$$
\int_{0}^{1} \frac{W(t)}{t^{p / r+1}} d t<\infty
$$

Then,

$$
\begin{aligned}
I_{1}^{p} & =\int_{0}^{\infty} W\left(\int_{\{x \in 3 B:|T(f)(x)|>t\}} u(x) d x\right) t^{p-1} d t \\
& \lesssim W(u(3 B))+\int_{1}^{\infty} W\left(\int_{\{x \in 3 B:|T(f)(x)|>t\}} u(x) d x\right) t^{p-1} d t .
\end{aligned}
$$

Since the condition $u \in A_{\infty}$ implies that $u \in A_{r}$, we get the classical estimate $T: L^{r}(u) \rightarrow L^{r, \infty}(u)$. Hence, for every $t>0$,

$$
\int_{\{x \in 3 B:|T(f)(x)|>t\}} u(x) d x \lesssim \int_{\left\{x \in \mathbb{R}^{n}:|T(f)(x)|>t\right\}} u(x) d x \lesssim \frac{\|f\|_{L^{r}(u)}^{r}}{t^{r}}
$$

and using that $W \in \Delta_{2}$,

$$
\begin{aligned}
& \int_{1}^{\infty} W\left(\int_{\{x \in 3 B:|T(f)(x)|>t\}} u(x) d x\right) t^{p-1} d t \lesssim \int_{1}^{\infty} W\left(\frac{\|f\|_{L^{r}(u)}^{r}}{t^{r}}\right) t^{p-1} d t \\
\lesssim & \int_{1}^{\infty} W\left(\frac{1}{t^{r}}\right) t^{p-1} d t \approx \int_{0}^{1} \frac{W(t)}{t^{p / r+1}} d t<\infty .
\end{aligned}
$$

Proof of Theorem 1.2. First, let $b$ be a bounded function and $f$ be a bounded function with compact support. Let $0<\beta<\varepsilon<1$. Since $T$ is bounded on $\Lambda_{u}^{p}(w)$ by Theorem 1.1, Tf lies in $\Lambda_{u}^{p}(w)$ and thus $b T(f)$ lies in $\Lambda_{u}^{p}(w)$ as well. Likewise $T(b f)$ lies in $\Lambda_{u}^{p}(w)$. Hence $\|[b, T](f)\|_{\Lambda_{u}^{p}(w)}<\infty$ and thus $\left\|M_{\beta}[b, T](f)\right\|_{\Lambda_{u}^{p}(w)}<\infty$, by the assumption $w \in B_{p}(u)$. Then, Lemma 2.6, Lemma 2.5, the condition $w \in B_{p}(u)$, and Theorem 1.1 lead us to

$$
\begin{aligned}
\|[b, T](f)\|_{\Lambda_{u}^{p}(w)} & \leq\left\|M_{\beta}[b, T](f)\right\|_{\Lambda_{u}^{p}(w)} \lesssim\left\|M_{\beta}^{\sharp}[b, T](f)\right\|_{\Lambda_{u}^{p}(w)} \\
& \lesssim\|b\|_{B M O}\left(\left\|M_{\varepsilon}(T(f))\right\|_{\Lambda_{u}^{p}(w)}+\left\|M^{2}(f)\right\|_{\Lambda_{u}^{p}(w)}\right) \\
& \lesssim\|b\|_{B M O}\left(\|T(f)\|_{\Lambda_{u}^{p}(w)}+\|f\|_{\Lambda_{u}^{p}(w)}\right) \lesssim\|b\|_{B M O}\|f\|_{\Lambda_{u}^{p}(w)} .
\end{aligned}
$$


In general, if $b$ is any function in $B M O$, define

$$
b_{N}(x)= \begin{cases}N, & \text { if } b(x)>N, \\ b(x), & \text { if }|b(x)| \leq N \\ -N, & \text { if } b(x)<-N\end{cases}
$$

It is obvious that $b_{N} \rightarrow b$ pointwisely and $\left\|b_{N}\right\|_{B M O} \leq\|b\|_{B M O}([10$, Exercise 3.1.4]). The Lebesgue dominated convergence theorem gives that $b_{N} \rightarrow b$ in $L^{2}(\operatorname{supp}(f))$. Hence $b_{k} f \rightarrow b f$ in $L^{2}\left(\mathbb{R}^{n}\right)$ and $T\left(b_{k} f\right) \rightarrow T(b f)$ in $L^{2}\left(\mathbb{R}^{n}\right)$, by the boundedness of $T$ on $L^{2}\left(\mathbb{R}^{n}\right)$. Thus, we deduce that there exists a subsequence of integers $k_{j}$, for which $T\left(b_{k_{j}} f\right) \rightarrow T(b f)$, a.e. For this subsequence, we obtain $\left[b_{k_{j}}, T\right](f) \rightarrow[b, T](f)$ a.e. Now, by Fatou's lemma in the weighted Lorentz spaces $\Lambda_{u}^{p}(w)$,

$$
\begin{aligned}
\|[b, T](f)\|_{\Lambda_{u}^{p}(w)} & \leq \liminf _{j \rightarrow \infty}\left\|\left[b_{k_{j}}, T\right](f)\right\|_{\Lambda_{u}^{p}(w)} \\
& \lesssim \liminf _{j \rightarrow \infty}\left\|b_{k_{j}}\right\|_{B M O}\|f\|_{\Lambda_{u}^{p}(w)} \lesssim\|b\|_{B M O}\|f\|_{\Lambda_{u}^{p}(w)} .
\end{aligned}
$$

\section{REFERENCES}

[1] E. Agora, M. J. Carro, and J. Soria, Characterization of the weak-type boundedness of the Hilbert transform on weighted Lorentz spaces, J. Fourier Anal. Appl. 19 (2013), 712-730.

[2] J. Alvarez and C. Pérez, Estimates with $A_{\infty}$ weights for various singular integral operators, Boll. Unione Mat. ital. 8 (1994), 123-133.

[3] M. A. Ariño and B. Muckenhoupt, Maximal functions on classical Lorentz spaces and Hardy's inequality with weights for nonincreasing functions, Trans. Amer. Math. Soc. 320 (1990), $727-735$.

[4] C. Bennett and R. Sharpley, Interpolation of Operators, Academic Press, 1988.

[5] M. J. Carro, J. A. Raposo, and J. Soria, Recent Developments in the Theory of Lorentz Spaces and Weighted Inequalities, Mem. Amer. Math. Soc. 187 (2007).

[6] M. J. Carro and J. Soria, Weighted Lorentz spaces and the Hardy operator, J. Funct. Anal. 112 (1993), no. 2, 480-494.

[7] R. R. Coifman and C. Fefferman, Weighted norm inequalities for maximal functions and singular integrals, Studia Math. 51 (1974), 241-250.

[8] R. R. Coifman, R. Rochberg, and G. Weiss, Factorization theorems for Hardy spaces in several variables, Ann. of Math. 103 (1976), 611-635.

[9] J. Duoandikoetxea, Weight for maximal functions and singular integrals, NCTH Summer School on Harmonic Analysis in Taiwan, 2005.

[10] L. Grafakos, Modern Fourier Analysis, 3rd edition, Graduate Texts in Math. 250, Springer, New York, 2014.

[11] R. A. Hunt, B. Muckenhoupt, and R. L. Wheeden, Weighted norm inequalities for the conjugate function and Hilbert transform, Trans. Amer. Math. Soc. 176 (1973), 227-251.

[12] H. L. Li and J. C. Chen, Convolutions, tensor products and multipliers of the Orlicz-Lorentz Spaces, Chin. Ann. Math. 36B (2015), 467-484.

[13] G. G. Lorentz, Some new functional spaces, Ann. of Math. 51 (1950), 37-55.

[14] B. Muckenhoupt, Weighted norm inequalities for the Hardy maximal function, Trans. Amer. Math. Soc. 165 (1972), 207-226.

[15] C. J. Neugebauer, Some classical operators on Lorentz spaces, Forum Math. 4 (1992), 135-146.

[16] C. Pérez, Endpoint estimates for commutators of singular integral operators, J. Funct. Anal. 128 (1995), 163-185.

[17] E. Sawyer, Boundedness of classical operators on classical Lorentz spaces, Studia Math. 96 (1990), 145-158.

[18] E. M. Stein and G. Weiss, Introduction to Fourier Analysis on Euclidean Spaces, Princeton University Press, 1971. 
Department of Analysis and Applied Mathematics, Complutense University of MADRID, 28040 MADRID, SPAIN.

Email address: mjcarro@ucm.es

Department of Mathematics, Zhejiang International Studies University, HangZHOU, 310012, CHINA.

Email address: honglli@126.com

Interdisciplinary Mathematics Institute (IMI), Department of Analysis and Applied Mathematics, Complutense University of Madrid, 28040 Madrid, Spain.

Email address: javier.soria@ucm.es

Department of Mathematics, Zhejiang University of Science and Technology, Hangzhou, 310023, China.

Email address: qxsun@126.com 